\title{
An Approximate Algorithm for the Minimal Cost Gateways Location, Capacity and Flow Assignment in Two-Level Hierarchical Wide Area Networks
}

\author{
Przemyslaw Ryba and Andrzej Kasprzak \\ Wroclaw University of Technology, Chair of Systems and Computer Networks \\ Wybrzeze Wyspianskiego 27, 50-370 Wroclaw, Poland \\ \{przemyslaw.ryba, andrzej.kasprzak\}@pwr.wroc.pl
}

\begin{abstract}
In the paper the problem of designing two-level hierarchical structure of wide area network is considered. The goal is to select gateways location, channel capacities and flow routes in order to minimize total cost of leasing capacities of channels of 2nd level network, subject to delay constraint. An approximate algorithm is proposed. Some results following from computational experiment are reported.
\end{abstract}

\section{Introduction}

Process of designing large wide area networks (WAN) containing hundreds of hosts and communication links is very difficult and demanding task. Conventional design procedures are suitable for small and moderate-sized networks. Unfortunately, when applied directly to large networks, they become very costly (from computational point of view) and sometimes infeasible. Problems with computational cost of huge wide area network design can be alleviated by building WAN as a hierarchical network [1]. In hierarchical networks, nodes are clustered using some nearness measures like geographical distance, traffic and reliability requirements. Communication networks of each cluster (1st level network) can be designed separately. In each cluster special communication nodes (gateways) are chosen. Function of gateways is to handle the traffic between nodes from the cluster they are located and nodes from other clusters. Gateways, with channels connecting them, form 2nd level network. Two-level networks can be grouped in 3rd level clusters and so on. Traffic between nodes in the same cluster uses paths contained in local communication network. Traffic between nodes in different 1 st level networks is first sent to local gateway, then via 2 nd level network of gateways is sent to gateway located in destination 1st level network to finally reach the destination node. Example of structure of hierarchical wide area network is presented in the Fig. 1.

In this paper problem of designing the two-level hierarchical wide area network connecting existing networks in order to minimize total cost of leasing capacities of channels of 2nd level network subject to delay constraint is considered. The Gateways Location, Capacity and Flow Assignment problem with cost criterion is formulated as follows: 
given: topology of 1st level networks and 2nd level network, sets of potential gateways locations, set of potential 2nd level network channels capacities and costs (i.e. discrete cost-capacity function), traffic requirements,

minimize: leasing cost of channel capacities of 2nd level network,

over: $\quad$ gateways locations, 2nd level network channel capacities, multicommodity flow (i.e. routing),

subject to: multicommodity flow constraints, channel capacity constraints, delay constraint in hierarchical network.

Discrete cost-capacity function considered here is most important from the practical point of view for the reason that channels capacities can be chosen from the sequence defined by ITU-T (International Telecommunication Union - Telecommunication Standardization Section) recommendations. Such formulated problem is NP-complete as more general than the CFA problem with discrete cost-capacity function which is NP-complete $[2,3]$.

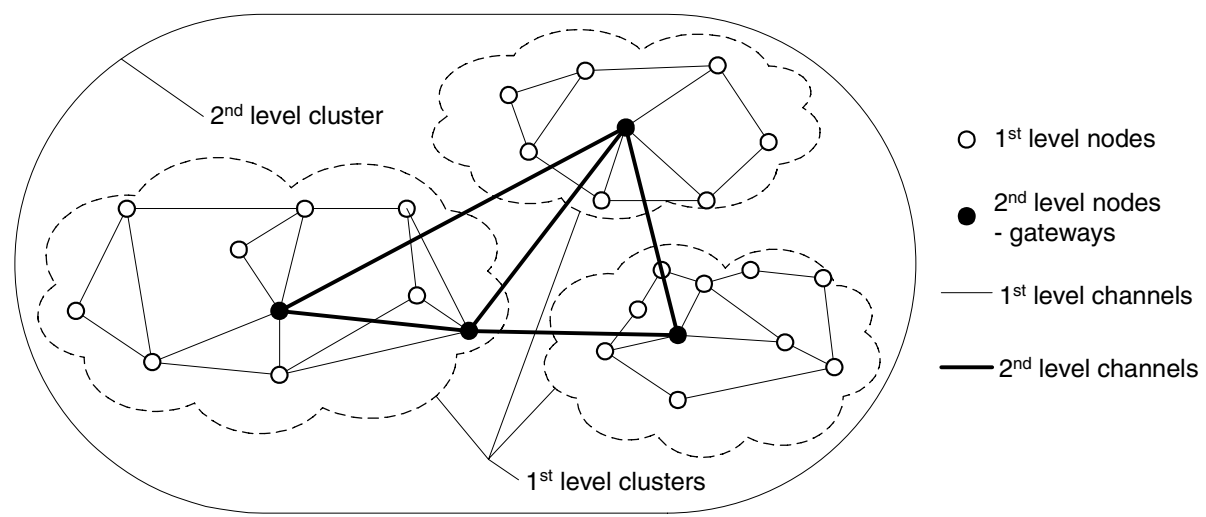

Fig. 1. Structure of the two-level hierarchical wide area network

Some algorithms for hierarchical network design can be found in $[4,5]$. However, problem presented in [5] is limited to tree topology of hierarchical network. Algorithm for router location minimizing total cost of the network without constraints on quality indices and without assigning capacities to channels is presented in the paper [4]. Also, in algorithm for interconnecting two WANs presented in [6] assigning capacities to channels connecting the networks is not considered.

The problems considered in the literature presented above, do not take into account that the gateways location problem and capacity assignment problem should be considered simultaneously. Solving this problem is important from practical point of view due to the fact that it results in significant reduction of network exploitation cost. This paper joins problem of locating gateways in hierarchical wide area network with capacity assignment problem to minimize network cost and to satisfy quality demands. Thus, problem considered in the paper is more general and more important from practical point of view than problems, which can be found in the literature. 


\section{Problem Formulation}

Consider a hierarchical WAN consisting of $K$ networks on 1 st level of hierarchy, each denoted by $S_{1}^{l}, l=1, \ldots, K$, and one 2 nd level network denoted by $S_{2}$. Let $N_{1}^{l}$ be set of nodes and $L_{1}^{l}$ set of channels of 1 st level network $S_{1}^{l}$. Let $n$ be the total number of nodes in hierarchical network. Set of nodes $N_{2}$ consists of selected gateways and $L_{2}$ is set of channels connecting them. Let $m$ be number of channels in 2 nd level of hierarchical WAN and let $p$ be total number of channels. For each channel in 2nd level network capacity must be chosen from the set of available capacities $C^{i}=\left\{c_{1}^{i}, \ldots, c_{s(i)}^{i}\right\}$. Capacities in each set $C^{i}$ are ordered in the following way: $c_{1}^{i}>c_{2}^{i}>\ldots>c_{s(i)}^{i}$. Let $d_{k}^{i}$ be the cost of leasing capacity $c_{k}^{i}$ for channel $i$.

Let $x_{k}^{i}$ be the discrete variable for choosing one of available capacities for channel $i$ defined as follows: $x_{k}^{i}=1$, if the capacity $c_{k}^{i}$ is assigned to channel $i$ and $x_{k}^{i}=0$ otherwise. Since exactly one capacity from the set $C^{i}$ must be chosen for channel $i$, the following condition must be satisfied:

$$
\sum_{k=1}^{s(i)} x_{k}^{i}=1 \text { for } i=1, \ldots, m
$$

Let denote by $H^{l}$ set of gateways to place in network $S_{1}^{l}$ and by $J_{g}$ set of possible locations for gateway $g$. Let $y_{a}^{g}$ be the discrete variable for choosing one of available locations for gateway $g$ defined as follows: $y_{a}^{g}=1$, if gateway $g$ is located in node $a$ and $y_{a}^{g}=0$, otherwise. Each gateway must be placed in exactly one node, thus it is required to satisfy following condition:

$$
\sum_{a \in J_{g}} y_{a}^{g}=1, g \in H^{l}, l=1, \ldots, K
$$

The cost $d_{k}^{i}$ of leasing capacity $c_{k}^{i}$ for channel $i$ is defined as follows:

$$
d_{k}^{i}=\sum_{a \in J_{g_{1}}} \sum_{b \in J_{g_{2}}} y_{a}^{g_{1}} y_{b}^{g_{2}} d_{a b}^{i k}
$$

where $g_{1}$ and $g_{2}$ are gateways adjacent to channel $i, d_{a b}^{i k}$ is cost of leasing capacity $c_{k}^{i}$ of channel $i$ between nodes $a$ and $b$, which are possible locations of gateways $g_{1}$ and $g_{2}$ respectively.

Let $X_{r}^{\prime}$ be the permutation of values of variables $x_{k}^{i}, i=1, \ldots, m$ for which the condition (1) is satisfied, and let $X_{r}$ be the set of variables which are equal to 1 in $X_{r}^{\prime}$. Similarly, let $Y_{r}^{\prime}$ be the permutation of values of all variables $y_{a}^{g}$ for which condition (2) is satisfied and let $Y_{r}$ be the set of variables which are equal to 1 in $Y_{r}^{\prime}$. The pair of sets $\left(X_{r}, Y_{r}\right)$ is called a selection. Each selection $\left(X_{r}, Y_{r}\right)$ 
determines locations of gateways and channels capacities in the 2nd level of hierarchical WAN. Let $\Re$ be the family of all selections.

Let $d\left(X_{r}, Y_{r}\right)$ be the total cost of leasing capacities of channels of 2nd level network of hierarchical network, in which values of channels capacities are given by $X_{r}$ and locations of gateways are given by $Y_{r}$.

$$
d\left(X_{r}, Y_{r}\right)=\sum_{x_{k}^{i} \in X_{r}} x_{k}^{i} d_{k}^{i}
$$

Then, the considered gateway location, flow and capacity assignment problem in hierarchical wide area network can be formulated as follows:

$$
\min _{\left(X_{r}, Y_{r}\right)} d\left(X_{r}, Y_{r}\right)
$$

subject to:

$$
\begin{gathered}
\left(X_{r}, Y_{r}\right) \in \Re \\
T\left(X_{r}, Y_{r}\right) \leq T^{\max }
\end{gathered}
$$

where $T\left(X_{r}, Y_{r}\right)$ denotes total average delay per packet in hierarchical network, given by Kleinrock's formula [7] and $T^{\max }$ its maximal acceptable value of that delay.

\section{Algorithm}

In this chapter we present an approximate algorithm for gateways location and capacity assignment minimizing cost of leasing capacities of channels of 2nd level network, subject to constraint on maximal admissible value of average delay per packet in hierarchical network.

Initially set $Y_{1}$ is calculated in such a way that total cost of leasing channels of 2nd level network is minimal. All channel capacities are set to maximal available values. If for such constructed network constraint (7) is violated, then one of gateways location is changed. Choice of gateway to change location is performed using exchange operation. After each change of gateway location new value of average delay per packet is compared with one already found. Location with higher value of average delay per packet is abandoned and removed from set of potential gateway locations. Operation is repeated until constraint (7) is satisfied. After that, capacities of selected channels is decreased to obtain network with lower leasing cost satisfying constraint (7) and value $d\left(X_{r}, Y_{r}\right)$ is compared with already found best solution $d^{*}$. If current cost of leasing channels capacities is lower, then $d^{*}$ is updated. When constraint (7) is violated then operation of changing gateway locations is repeated. Algorithm terminates, when, for each gateway, the set of possible locations is empty or there is no channel which capacity can be decreased. Algorithm finds heuristic solution after no more than $\left(\sum_{l=1}^{K} \sum_{g \in H^{l}}\left|J^{g}\right|\right)\left(\sum_{i=1}^{m} s(i)\right)$ iterations. 


\subsection{Exchange Operations}

The purpose of exchange operation is to find a pair of variables $x_{k}^{i}$ or variables $y_{a}^{g}$ for substitution to generate a network with the least possible value of criterion (4). To take into account the constraint (7) we propose the auxiliary local criterion: $d\left(X_{r}, Y_{r}\right)+\alpha \cdot T\left(X_{r}, Y_{r}\right)$, where coefficient $\alpha$ converts value of average packets delay to cost expressed in [€/month]. Choice of variables $x_{k}^{i}$ and $x_{j}^{i}$ to exchange is made using modified criterion $\Delta_{k j}^{i}$ usually used in classical CFA problem, formulated as follow $[2,8]$ :

$$
\Delta_{k j}^{i}=\left\{\begin{array}{l}
\frac{\alpha}{\gamma}\left(\frac{f_{i}}{c_{k}^{i}-f_{i}}-\frac{f_{i}}{c_{j}^{i}-f_{i}}\right)+\left(d_{k}^{i}-d_{j}^{i}\right) \quad \text { for } f_{i}<c^{i} \\
\infty \quad \text { otherwise }
\end{array}\right.
$$

Choice of variables $y_{a}^{g}$ and $y_{b}^{g}$ to exchange may be evaluated by criterion $\delta_{a b}^{g}$.

$$
\delta_{a b}^{g}=\left\{\begin{array}{l}
\frac{\alpha}{\gamma} \sum_{i \in L_{1}^{l}}\left(\frac{\tilde{f}_{i}}{c^{i}-\tilde{f}_{i}}-\frac{f_{i}}{c^{i}-f_{i}}\right)+\sum_{i:\left\{g, g_{2}\right\rangle \in V^{g}}\left(d_{e b}^{i j}-d_{e a}^{i j}\right) \text { for } \tilde{f}_{i} \leq c^{i} \\
\infty \quad \text { otherwise }
\end{array}\right.
$$

The second term of the sum estimates change of the leasing cost of channels adjacent to the gateway $g$ of which location changes. The flow $\tilde{\mathbf{f}}$ is constructed as follows: the flow from all nodes in network $S_{1}^{l}$ sent through gateway located in node $a$ is moved from the routes leading to node $a$ to the routes leading to node $b$. If the conditions $\tilde{f}_{i} \leq c^{i}$ are satisfied for every channel in the network $S_{1}^{l}$ the flow $\tilde{\mathbf{f}}$ is feasible.

To exchange should be chosen the pair of variables for which the value of criterion $\delta_{a b}^{g}$ or $\Delta_{k j}^{i}$ is minimal.

\subsection{Calculation Scheme of the Approximate Algorithm}

Let $J_{g}^{r}$ be the set of possible locations for gateway $g$ in r-th iteration of the approximate algorithm. Let $J_{g}^{1}=J_{g}$ for each gateway $g$. Let $Y_{1}=\varnothing, d^{*}=\infty, r=1$.

Step 1. Perform $x_{1}^{i}=1$ for $i=1, \ldots, m$. Compute $Y_{1}$, such that $d\left(X_{1}, Y_{1}\right)=\min _{Y_{r}} d\left(X_{1}, Y_{r}\right)$

Step 2. Compute flow $\mathbf{f}$ using FD method [9], next compute $T\left(X_{r}, Y_{r}\right)$.

If $T\left(X_{r}, Y_{r}\right)>T^{\max }$ then go to step 3. Otherwise go to step 4 . 
Step 3. If $J_{g}^{r}=\varnothing$ for every $g$ then go to step 6. Otherwise select the pair of variables $y_{a}^{g} \in Y_{r}$ and $y_{b}^{g}: b \in J_{g}^{r}$ for which with the value of expression (9) is the greatest. Perform $Y_{r+1}=\left(Y_{r}-\left\{y_{a}^{g}\right\}\right) \cup\left\{y_{b}^{g}\right\}$ Next compute $T\left(X_{r}, Y_{r+1}\right)$.

If $T\left(X_{r}, Y_{r+1}\right)>T\left(X_{r}, Y_{r}\right)$ then perform $J_{g}^{r}=J_{g}^{r}-\{b\}$ and go to step 2 .

Otherwise perform $J_{g}^{r+1}=J_{g}^{r}-\{a\}$ and $r=r+1$. Next go to step 2 .

Step 4. If $d\left(X_{r}, Y_{r}\right)<d^{*}$ then perform $d^{*}=d\left(X_{r}, Y_{r}\right)$ and $\left(X_{*}, Y_{*}\right)=\left(X_{r}, Y_{r}\right)$.

Step 5. If $k=s(i)$ for every $x_{k}^{i} \in X_{r}$ then go to step 6. Otherwise select variable $x_{k}^{i} \in X_{r}$, such that $k \leq s(i)-1$, with greatest value of expression (8) and perform $X_{r+1}=\left(X_{r}-\left\{x_{k}^{i}\right\}\right) \cup\left\{x_{k+1}^{i}\right\}$ and $r=r+1$. Go to step 2 .

Step 6. Algorithm terminates. If $d^{*}=\infty$ then problem has no solution. Otherwise the selection $\left(X_{*}, Y_{*}\right)$ associated with current value $d^{*}$ is the near-optimal solution satisfying the constraints (6) and (7).

\section{Computational Results}

The presented approximate algorithm was implemented in $\mathrm{C}++$ code. Extensive numerical experiments have been performed with this algorithm for many different hierarchical network topologies and for many possible gateways number and locations. The experiments were conducted with two main purposes in mind: first, to examine the impact of various parameters on solutions to find properties of the problem (5-7) important from practical point of view and second: to test the computational efficiency of proposed algorithm.

The dependence of the cost of leasing channel capacities $d$ on maximal acceptable value of average delay per packet in hierarchical network $T^{\max }$ has been examined. In the Fig. 2 the typical dependence of $d$ on the value $T^{\max }$ is presented for different

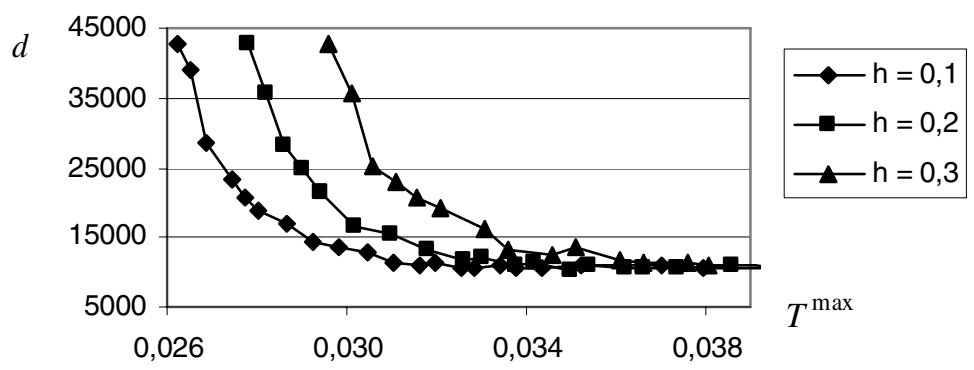

Fig. 2. The dependence of criterion function $d$ on value $T^{\max }$ 
values of the average packet rates from external sources transmitted between each pair of nodes of the hierarchical network, which are denoted by $h$. It can be observed that the dependence of leasing cost of channel capacities $d$ on $T^{\max }$ is decreasing function and that there exists such value $\hat{T}^{\max }$, that the problem (5-7) has the same solution for each $T^{\max }$ greater or equal to $\hat{T}^{\max }$.

Let $G$ be the number of gateways, which must be allocated in some 1st level network of the hierarchical WAN. It has been examinated how number of gateways $G$ influences on the criterion function $d$. In the Fig. 3 typical dependence of leasing cost of channel capacities $d$ on number of gateways $G$ is presented.

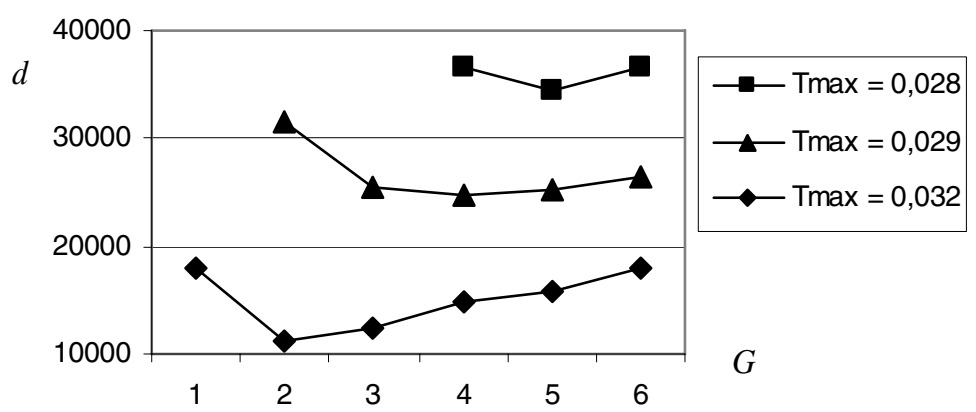

Fig. 3. The dependence of criterion function $d$ on number of gateways $G$

Fig. 3 indicates that higher quality demands (lower values of constraint $T^{\max }$ ) imply allocating higher number of gateways. For small number of gateways feasible solution cannot be found.. It can be also observed, that there exists such value of $G$ for which the function $d(G)$ is minimal. Number of gateways $G$, for which value $d$ is minimal, is higher for tighter constraint on $T^{\max }$.

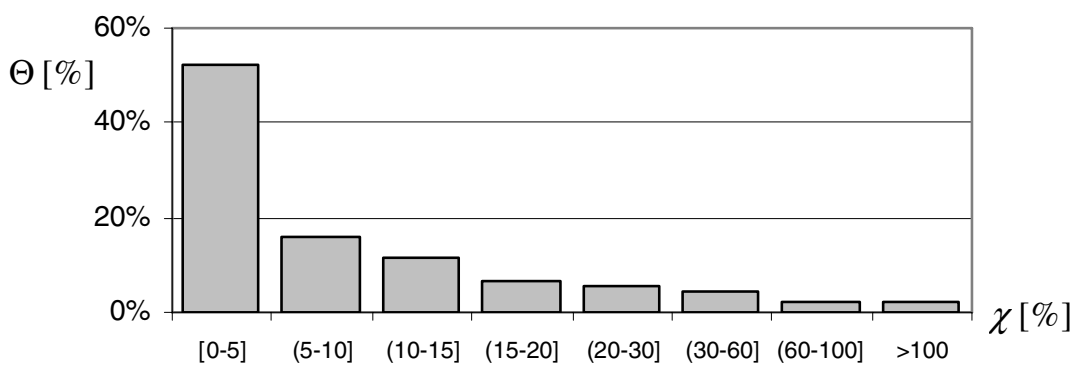

Fig. 4. The distance between obtained solutions and optimal solutions

Let $d^{a p p}$ be the solution obtained by approximate algorithm and let $d^{\text {opt }}$ be the optimal value of the problem (5-7). Let $\chi$ be the distance between approximate and optimal solutions: $\chi=\left|d^{\text {app }}-d^{\text {opt }}\right| / d^{\text {opt }} \cdot 100 \%$. The value $\chi$ shows how 
the results obtained using the approximate algorithm are worse than the optimal solution.

Let

$$
\Theta[a, b]=\frac{\text { number of solutions for which } \chi \in[a, b]}{\text { number of all solutions }} \cdot 100 \%
$$

denotes the fraction of solutions obtained from the approximate algorithm which are greater than optimal solutions between $a \%$ and $b \%$. The dependence $\Theta$ on divisions $[0 \%-5 \%],(5 \%-10 \%),[10 \%-15 \%)$, etc. is shown in the Fig. 4.

\section{Conclusions}

The approximate algorithm for solving the gateway location and network topology assignment problem in hierarchical network to minimize total cost of leasing channel capacities is presented. The considered problem is more general than the similar problems presented in the literature. It follows from computational experiments (Fig. 4) that more than 50\% approximate solutions differ from optimal solutions at most 5\%. Moreover, we noticed that the cost of the hierarchical WAN depends on the number of gateways. The presented approximate algorithm may be used whenever an optimal solution is not necessary and for large hierarchical networks.

This work was supported by a research project of The Polish State Committee for Scientific Research in 2005-2007.

\section{References}

1. Kleinrock L., Kamoun F.: Optimal Clustering Structures for Hierarchical Topological Network Design of Large Computer Networks. Networks 10 (1980) 221-248

2. Kasprzak, A.: Topological Design of the Wide Area Networks. Wroclaw University of Technology Press, Wroclaw (2001)

3. Markowski M., Kasprzak A.: An exact algorithm for host allocation, capacity and flow assignment problem in WAN. WITASI 2002, Kluwer Academic Publ. (2002) 73-82

4. Liu Z., Gu Y., Medhi D.: On Optimal Location of Switches/Routers and Interconnection. Technical Report, University of Missouri-Kansas City (1998)

5. Saha D. and Mukherjee A.: On the multidensity gateway location problem for multilevel high speed network. Computer Communications 20 (1997) 576-585

6. Liang S.C., Yee J.R.: Locating Internet Gateways to Minimize Nonlinear Congestion Costs. IEEE Transactions On Communications 42, (1994), 2740-50

7. Fratta, L., Gerla, M., Kleinrock, L.: The Flow Deviation Method: an Approach to Storeand-Forward Communication Network Design. Networks 3 (1973) 97-133

8. Markowski M., Kasprzak A.: The Web Replica Allocation and Topology Assignment Problem in Wide Area Networks: Algorithms and Computational Results. Lecture Notes in Computer Science 3483 (2005) 772-781

9. Walkowiak K.: A New Method of Primary Routes Selection for Local Restoration, Lectures Notes in Computer Science 3042 (2004) 1024-1035 